\title{
Characterizing InGaAs/GaAs quantum dots using low-kV FESEM imaging and EDS analysis at the nanometer scale
}

\author{
Fang Zhou ${ }^{1}$, Luyang $\operatorname{Han}^{1}$, Simon Burgess ${ }^{2}$, and Xiaobing $\mathrm{Li}^{2}$. \\ 1. Carl Zeiss Microscopy GmbH, Carl-Zeiss-Straße 22, 73447 Oberkochen, Germany \\ 2. Oxford Instruments NanoAnalysis, Halifax Road, High Wycombe, Bucks HP12 3SE, UK
}

Semiconductor quantum dots (QDs) have potential applications as single photon sources [1] or as quantum bits (qubits) in the field of quantum information processing [2]. The electronic properties or the energy band structures of a QD depend strongly on its dimension, shape and composition which should be well engineered and characterized by means of surface sensitive SEM imaging and material sensitive analysis. InGaAs/GaAs quantum dot heterostructures in this study have a lens shape with a base diameter of about $20 \mathrm{~nm}$ and height of about $3 \mathrm{~nm}$ which can be imaged using a high resolution field emission scanning electron microscope (FESEM) operated at low-kV. The In-concentration and Indistribution in a QD are strongly correlated with the quality of the QD. However, traditional EDS analysis, which typically operates at 10 to $15 \mathrm{kV}$, cannot provide elemental characterization on such small feature like QD at the nanometer scale due to the huge scattering volume in its bulk substrate.

In this work, we present a unique combination of Zeiss GeminiSEM 500 and the X-Max Extreme EDS system from Oxford Instruments which enables characterization of InGaAs/GaAs QD heterostructure’s morphology and composition simultaneously.

Fig.1a shows InGaAs QDs on the surface of a GaAs substrate using the Zeiss GeminiSEM 500. This ultra-high resolution FESEM offers exciting new capabilities for investigating smaller nano-structures, interfaces and surfaces of a large variety of sample types [3]. The unique inlens SE and EsB detectors provide extremely surface-sensitive high resolution morphological and compositional contrast, optimized to operate at 1-3 keV low beam energy. It must be noted that such small and thin features can only be imaged with high contrast at low beam energy in the range of 1 to $3 \mathrm{keV}$. Thus, under this imaging condition only low energy X-rays are excited from the sample. With the windowless X-Max Extreme from Oxford Instruments, however, the low energy X-rays down to $50 \mathrm{eV}$ can be detected and analyzed. Fig.1b shows the point analysis of the InGaAs QD and the GaAs substrate, respectively. This implementation maintains high sensitivity and is able to verify the local In-concentration in such small features due to the differences in the spectra, notably at $366 \mathrm{eV}$. In addition, Fig.2 shows the elemental mapping of such QD heterostructures using $2 \mathrm{kV}$ beam energy. Such a map suggests that the QDs are preferentially oxidized.

References:

[1] P. Michler, A. Kiraz, C. Becher, W. V. Schoenfeld, P. M. Petroff, L. Zhang, E. Hu, and A. Imamoglu, Science 290, pp.2282, 2000

[2] G. Burkard, D. Loss, and D. P. DiVincenzo, Phys. Rev. B 59, pp.2070, 1999

[3] L. Maniguet, F. Roussel, R. Martin, E. Djurado, M.C. Steil, E. Bichaud, A. Le Goff, M. Holzinger, S. Cosnier, J.M. Chaix, C. Carry, Microscopy and Analysis, Nanotechnology Supplement Nov/Dec, pp.4, 2015 
In lens SE

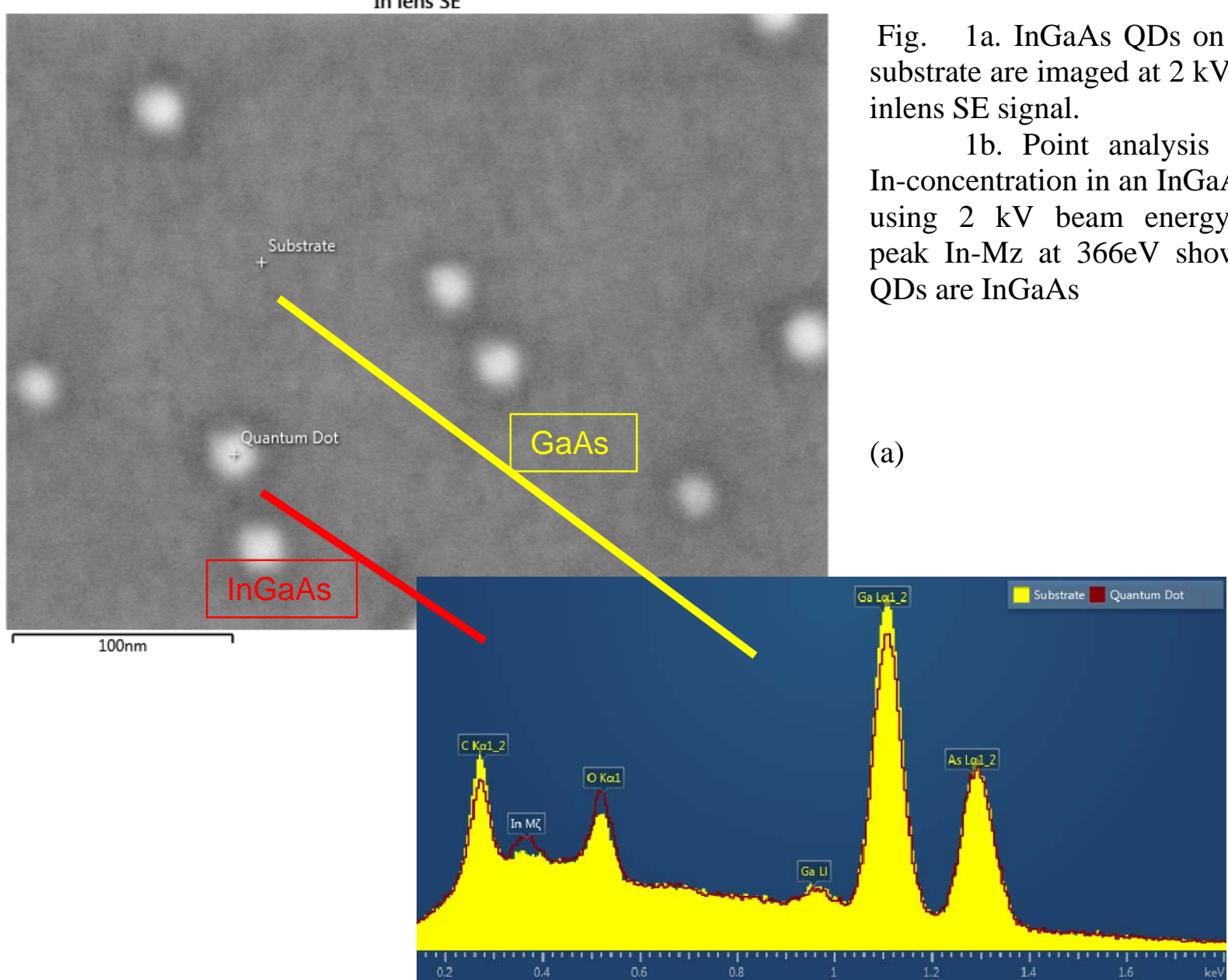

EDS Layered Image

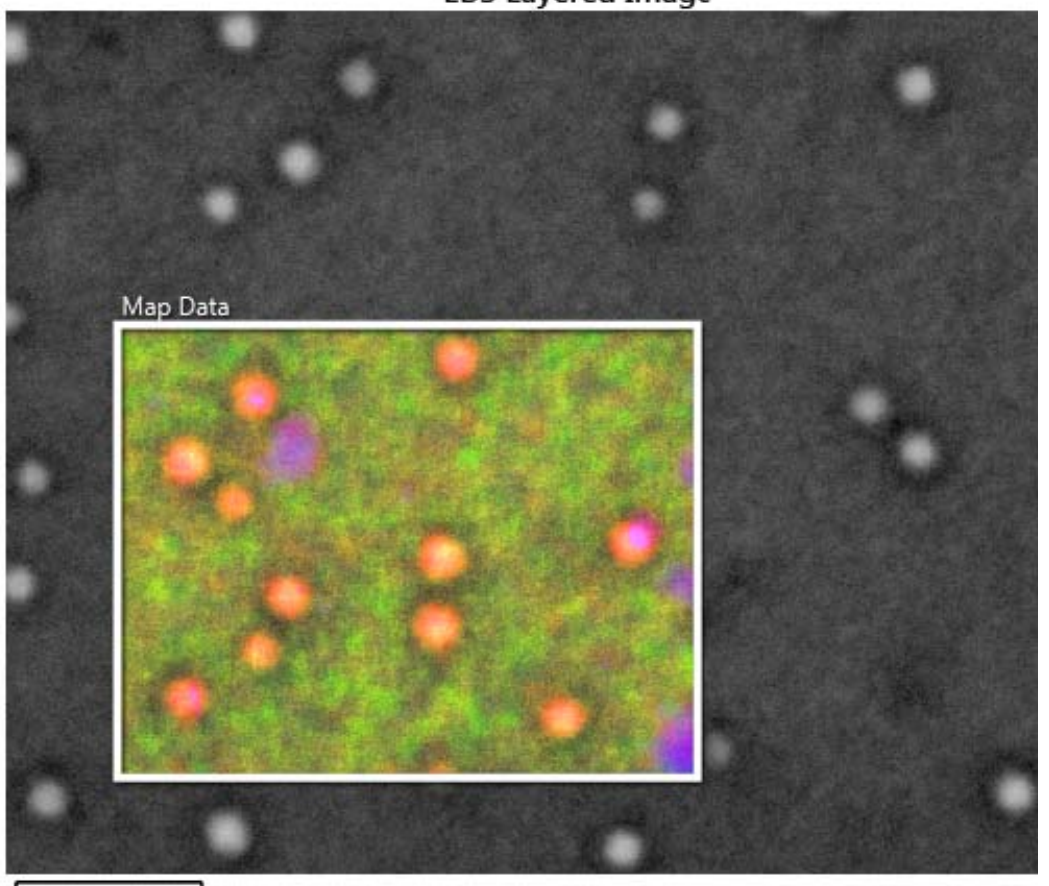

$100 \mathrm{~nm}$

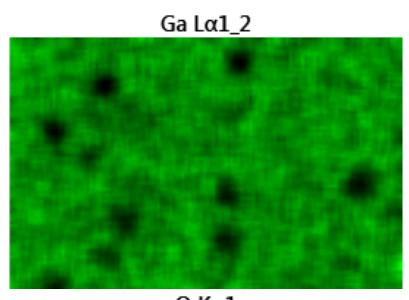

$\mathrm{OK} \alpha 1$

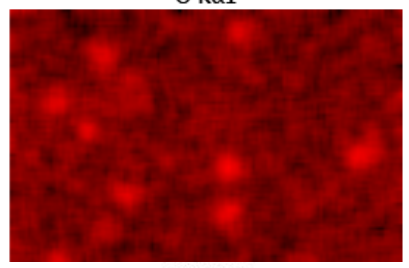

C K $\alpha 1 \_2$

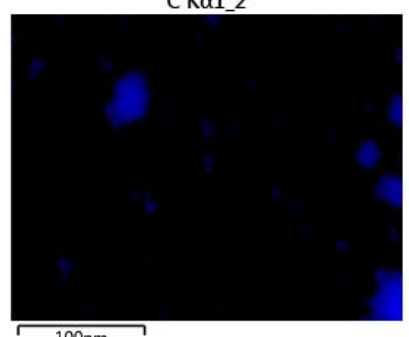

$100 \mathrm{~nm}$

Fig2. Element mapping at $2 \mathrm{kV}, 4,000 \mathrm{cps}$ for 20 mins. Quantum dots are preferentially oxidized. 